\title{
Prevalence of plasmid-mediated quinolone resistance qur genes in Central China
}

\author{
Xuan Cai, Congrong Li ${ }^{\star}$, Jun Huang and Yan Li \\ Department of Clinical Laboratory, Renmin Hospital of Wuhan University, Wuhan 430060, People's Republic of China. \\ Accepted 7 April, 2011

\begin{abstract}
A total of 179 Gram-negative bacteria strains collected in Wuhan from 2005 to 2006 were screened for qnrA, qnrB and qnrS genes by polymerase chain reaction (PCR). The qnrA genes were detected in $3.88 \%$ of Escherichia coli and 7.69\% of Enterobacter cloacae, whereas the qnrB genes were found in $6.20 \%$ of $E$. coli and $7.69 \%$ of E. cloacae. $2.33 \%$ of E. coli and $18.92 \%$ of Klebsiella pneumoniae were positive for the qnr gene in PCR. Minimal inhibitory concentrations (MICs) results of ciprofloxacin confirmed that qnrS gene could be present in isolates susceptible to quinolones. Furthermore, the results indicated that qnr genes were identified on transferable plasmids, and quinolone resistance can be transferred by bacterial conjugation.
\end{abstract}

Key words: qnr, gram-negative pathogens, plasmid-mediated.

\section{INTRODUCTION}

Quinolone resistance has traditionally been attributed to chromosomal mutations, such as DNA gyrase and topoisomerase IV, or activated efflux pumps. However, the plasmid-mediated quinolone resistance associated with gnr (now named qnrA1) in Klebsiella pneumoniae was firstly found from the United States in 1998 (Martinez-Martinez et al., 1998). The qnr gene encodes a 218-amino-acid protein. The purified qnr protein can protect DNA gyrase and topoisomerase IV activity in vitro. Furthermore, the qnr-plasmids are I type integron-associated plasmid and carry multiple resistance determinants providing resistance to several classes of antimicrobials including $\beta$-lactams, aminoglycosides and sulfamido (Martinez-Martinez et al., 1998; Tran et al., 2005). The qnr gene has already been reported in all populated continents except South America (Robicsek et al., 2006a). And in different geographical areas, the prevalence of qnr gene type was different. In Japan, qnrS gene was found in Shigella flexneri $2 \mathrm{~b}$, which showed $59 \%$ amino acid identity with the gnr gene (Hata et al.,

*Corresponding author. E-mail: congrongli33@hotmail.com. Tel: 86-27-88071553. Fax: 86-27-88071553.
2005). In India, qnrB was found in Citrobacter koseri with $40 \%$ amino acid identity with qnr (Jacoby et al., 2006). The present research was conducted to study the prevalence of $q n r A$, qnrB and $q n r S$ genes among clinical isolates of Gram negative bacteria in Hubei province of China.

\section{MATERIALS AND METHODS}

Clinical isolates

179 Gram negative bacteria strains (including 129 of Escherichia coli, 37 of K. pneumoniae and 13 of Enterobacter cloacae) were collected from June 2005 to February 2006 in Renmin Hospital of Wuhan University, a District teaching hospital with 1300 ward beds located in central China. All isolates were collected from individual patients who were hospitalized in various types of wards. Species identification was performed using Vitek 32 GNI cards. Additional strains used were E. coli C600Lac- SMR (resistance to streptomycin) as a recipient for conjugation.

\section{Susceptibility tests}

A disk diffusion susceptibility test was performed on Mueller-Hinton agar by a comparative method. Ampicillin, ampicillin/clavulanic acid, ceftazidime, cefotaxime, cefoxitin and imipenem disks were used. 
Minimal inhibitory concentrations (MICs) of the antibiotics were determined according to the Clinical and Laboratory Standards Institute (CLSI) 2007. E. coli ATCC25922 and Pseudomonas aeruginosa ATCC27853 were used as quality control reference strains. MICs of ciprofloxacin were determined by agar dilution assay.

\section{Screening for the qnr gene in clinical strains}

Screening was carried out by polymerase chain reaction (PCR) amplification of $q n r A, q n r B$ and $q n r S$. Bacteria isolates were transferred to TE buffer in Eppendorf tubes, and then boiled for 10 min. The supernatant was used as DNA templates for PCR. Primers used were as follows: for qnrA, 5'-TCAGCAAGAGGATTTCTCA-3' and 5'-GGCAGCACTATTA CTCCCA-3' to give a 516 bp product, and the PCR conditions were $94^{\circ} \mathrm{C}$ for $5 \mathrm{~min}, 94^{\circ} \mathrm{C}$ for $45 \mathrm{~s}, 48^{\circ} \mathrm{C}$ for $45 \mathrm{~s}$, and $72^{\circ} \mathrm{C}$ for $45 \mathrm{~s}$ and finally $72^{\circ} \mathrm{C}$ for $7 \mathrm{~min}$, with a cycle number of 30; for qnrB, 5'-GATCGTGAAAGCCAGAAAGG-3' and 5'-ACGATG CCTGGTAGTTGTCC-3' to give a 469 bp product, and for qnrS, 5'-ACGACATT CGTCAACTGCAA-3',5'-TAAATTGGCACCCTGTAGGC-3'to give a 417 bp product, and the PCR conditions were $94^{\circ} \mathrm{C}$ for $5 \mathrm{~min}, 94^{\circ} \mathrm{C}$ for $45 \mathrm{~s}, 53^{\circ} \mathrm{C}$ for $45 \mathrm{~s}$, and $72^{\circ} \mathrm{C}$ for $1 \mathrm{~min}$, with 32 cycles. Amplification products were detected by electrophoresis on a $2 \%$ agarose gel with ethidium bromide and photographed under UV light. All positive products were conformed by direct sequencing of PCR products.

\section{Conjugation experiments}

Conjugation experiments were carried out with E. coli C600LacSMR as the recipient. The recipients were selected on $\mathrm{M}-\mathrm{H}$ with streptomycin $(2000 \mathrm{mg} / \mathrm{L})$ and the donors were selected on $\mathrm{M}-\mathrm{H}$ with ciprofloxacin $(0.06 \mu \mathrm{g} / \mathrm{ml})$ (BioMérieux, France). Both recipients and donors were added to $2 \mathrm{ml}$ of fresh LB broth and incubated for16 to $24 \mathrm{~h}$. Culture of donor and recipient cells in logarithmic phase $(0.5 \mathrm{ml}$ each) were added to $4 \mathrm{ml}$ of fresh LB broth and incubated 16 to $24 \mathrm{~h}$ without shaking. Transconjugants were selected on MacConkey agar plates supplemented with streptomycin $(2000 \mathrm{mg} / \mathrm{L})$ and ciprofloxacin $(0.06 \mu \mathrm{g} / \mathrm{ml})$. To determine if qnr gene was co-transfered, PCR was done to detect qnr gene in transconjugants. Then products were checked by electrophoresis on a $1 \%$ agarose gel with ethidium bromide and photographed under UV light.

\section{Sequencing}

The products were purified with Qiaquick PCR purification kit (QIAGEN, USA). DNA products after amplification were sequenced with an Applied Biosystems sequencer. The nucleotide and deduced protein sequences were analyzed with software available over the Internet at the National Center for Biotechnology Information website (www.ncbi.nlm.nih.gov). The ClustalW program (www.infobiogen.fr) was used to align multiple protein sequences.

\section{RESULTS}

\section{Screening for the qnr gene and sequencing}

The qnr gene was detected in 25 (13.97\%) of 179 Gram negative bacteria (including 129 of E. coli, 37 of
K. pneumoniae and 13 of E. cloacae) by PCR (Table 1). Among the 179 isolates, the qnrA genes were present in $3.88 \%$ of $E$. coli and $7.69 \%$ of E. cloacae. The qnrB genes were present in $6.20 \%$ of $E$. coli and $7.69 \%$ of E. cloacae. The qnrS genes were present in $2.33 \%$ of $E$. coli and $18.92 \%$ of $K$. pneumoniae. We did not find qnrA and $q n r B$ genes in any of the $37 \mathrm{~K}$. pneunoniae and did not find qnrS gene in any of the $13 \mathrm{E}$. cloacae. Positive isolates were sequenced with an Applied Biosystems sequencer. All gnrA genes were of the qnrA1 allele, and E. coli 175 had a single nucleotide change (CTA $\rightarrow$ CTG at position 537) (A839G) without amino acid alteration. qnrB genes were of the qnrB4 and qnrB6 variants, and qnrS genes were of the qnrS1 allele.

\section{Susceptibility tests}

Antibiotic susceptibility testing by disk diffusion revealed that the 25 isolates were all susceptible to imipenem but resistant to most $\beta$-lactams, aminoglycosides and sulfamido, including cefotaxime, ceftazidime, aztreonam, amikacin and cefoxitin and so on. Among the MICs of all positive isolates (Tables 2, 3 and 4), 2 qnrA positive isolates and 4 qnrB positive isolates were susceptive to ciprofloxacin.

\section{Transfer of quinolone resistance}

Only 10 qnr positive isolates were found to transfer resistance to $E$. coli C600Lac-SMR, including 2 gnrA positive isolates, 4 gnrB and 4 qnrS positive isolates. The qnr genes from the transconjugants can be detected by PCR.

\section{DISCUSSION}

It has been more than 20 years since the fluoroquinolones drugs were introduced into China. Bacterial resistance to quinolones has increased markedly. Some of the national studies suggest that the fluoroquinolone resistance rates in E. coli were higher than previously estimated 50 to $60 \%$ (Li et al., 2001, 2003). In this study, the prevalence of qnr plasmid-mediated quinolone resistance has been first reported in Central China.

The $q n r$ genes were detected in 25 (13.97\%) of 179 Gram negative bacteria (including 129 of E. coli, 37 of K. pneumoniae and 13 of E. cloacae). There were 6 strains of qnrA genes, 9 strains of $q n r B$ genes and 10 strains of qnrS genes. Among the 179 isolates, the qnrA genes were present in $3.88 \%$ of $E$. coli and $7.69 \%$ of E. cloacae. The $q n r B$ genes were present in $6.20 \%$ of $E$. coli and $7.69 \%$ of $E$. cloacae. The qnrS genes were present in $2.33 \%$ of $E$. coli and $18.92 \%$ of $K$. pneumoniae. The rate 
Table 1. Prevalence of qnr genes in gram negative bacteria isolates.

\begin{tabular}{lccc}
\hline \multirow{2}{*}{ qnr } & \multicolumn{3}{c}{ No. of positive isolates/total no. of isolates (\%) } \\
\cline { 2 - 4 } & E. coli (\%) & E. cloacae & K. pneumoniae \\
\hline qnrA & $5 / 129(3.88)$ & $1 / 13(7.69 \%)$ & $0 / 37$ \\
qnrB & $8 / 129(6.20)$ & $1 / 13(7.69 \%)$ & $0 / 37$ \\
qnrS & $3 / 129(2.33)$ & $0 / 13$ & $7 / 37(18.92 \%)$ \\
Total & $16 / 129(12.40)$ & $2 / 13(15.38 \%)$ & $7 / 37(18.92 \%)$ \\
\hline
\end{tabular}

Table 2. MICs of qnrA positive isolates.

\begin{tabular}{lllcccc}
\hline & \multicolumn{7}{c}{ Positive isolates } \\
\hline Ciprofloxacin & 17 & 24 & 25 & 62 & 151 & 175 \\
MIC $(\mu \mathrm{g} / \mathrm{ml})$ & 16 & 16 & 64 & 8 & 1 & 0.25 \\
\hline
\end{tabular}

Table 3. MICs of qnrB positive isolates.

\begin{tabular}{llllcccccc}
\hline \multicolumn{10}{c}{ Positive isolates } \\
\hline Ciprofloxacin & 21 & 75 & 87 & 91 & 95 & 96 & 106 & 128 & 307 \\
$\mathrm{MIC}(\mu \mathrm{g} / \mathrm{ml})$ & 32 & 32 & 16 & 8 & 0.25 & 1 & 0.0625 & 16 & 0.0625 \\
\hline
\end{tabular}

Table 4. MICs of qnrS positive isolates.

\begin{tabular}{lcccccccccc}
\hline \multicolumn{10}{c}{ Positive isolates } \\
\hline Ciprofloxacin & 49 & 105 & 114 & 204 & 205 & 207 & 209 & 213 & 226 & 231 \\
$\mathrm{MIC}(\mu \mathrm{g} / \mathrm{ml})$ & 32 & 16 & 64 & 8 & 16 & 8 & 32 & 4 & 16 & 4 \\
\hline
\end{tabular}

of qnrA positive $E$. coli isolates is lower than that in Shanghai reports in China (Wang et al., 2003). A recent study in the United States found that qnrA and qnrB genes were detected in $15(14 \%)$ and $6(6 \%)$ of $106 \mathrm{~K}$. pneumoniae isolates (Robicsek et al., 2006b). But in our study, qnrA and qnrB genes were not detected in any of the $37 \mathrm{~K}$. pneunoniae. It is probably associated with the geographical distribution of qnr genes.

The antibiotic susceptibility test revealed that all of those 25 isolates were susceptible to imipenem but resistant to most $\beta$-lactams, aminoglycosides and sulfamido, including cefotaxime, ceftazidime, aztreonam, amikacin and cefoxitin and so on. However, as much to be emphasized in our study is that we find out qnr genes can also be found in susceptible organisms (may be due to lack of sufficient chromosomal mutations). 2 gnrA positive isolates and 4 qnrB positive isolates were susceptive to ciprofloxacin in our study. Among the 6 susceptive isolates, the E. coli 96 and E. cloacae 307 were also susceptive to ceftazidime. Some reports manifest that these organisms are likely clinically worrisome as resistant organisms (Tran et al., 2005). For under ongoing selective pressure, such organisms, in vitro, can readily develop resistance. For instance, Poirel et al. (2006) had reported a sensitive strain of $E$. coli harboring $q n r A$ with no classic quinolone resistance mutation, and it was found to develop chromosomal mutations and subsequent high-level resistance after 5 days of norfloxacin therapy (Poirel et al., 2006). Whether it is safe or not to use fluoroquinolone to treat qnr-bearing organisms susceptive 
to quinolones is a key issue to be resolved. Therefore, much more work is required to further define the proportion of clinical Enterobacteriaceae harboring such low-level resistance and the effect of these genes on clinical outcomes.

In conjugation tests, quinolone resistance was found to transfer respectively. But only 10 positive isolates were transferred successfully, including 2 qnrA positive isolates, 4 qnrB and 4 qnrS positive isolates. It is probably that the plasmids were lost or broken when they were transferred. In our study, we found that qnr genes could also be found in extended-spectrum beta-lactamase (ESBL)-producing isolates and the more associations should be studied in the future. The emergence of plasmid-determined quinolone resistance probably results in the rapid increase in bacterial resistance to quinolones in China. So it is necessary to study the prevalence qnr genes as well as the mechanism of qnr genes involved in quinolones resistance further in the future in China.

\section{REFERENCES}

Hata M, Suzuki M, Matsumoto M, Takahashi M, Sato K, Ibe S, Sakae K (2005). Cloning of a novel gene for quinolone resistance from a transferable plasmid in Shigella flexneri 2b. Antimicrob. Agents. Chemother., 49: 801-803.

Jacoby GA, Walsh KE, Mills DM, Walker VJ, Oh H, Robicsek A, Hooper DC (2006). qnrB, another plasmid-mediate gene for quinolone resistance. Antimicrob. Agents Chemother., 50: 1178-1182.
Li JT, Li Y, Wang J, China Bacterial Resistance Surveillance Study Group (2003). Surveillance on drug resistance of Gram-negative bacilli isolated from hospital acquired infections and community acquired infections (2000-2001). Zhonghua. Yi. Xue. Za. Zhi, 83: 1035-1045.

Li J, Weinstein AJ, Yang M (2001). China Bacterial Resistance Surveillance Study Group Surveillance of bacterial resistance in China (1998-1999). Zhonghua. Yi. Xue. Za. Zhi., 81: 8-16.

Martinez-Martinez L, Pascual A, Jacoby GA (1998). Quinolone resistance from a transferable plasmid. Lancet., 351: 797-799.

Minggui W, John HT, George AJ, Yingyuan Z, Fu W, David CH (2003). Plasmid-mediated quinolone resistance in clinical isolates of Escherichia coli from Shanghai, China. Antimicrob. Agents. Chemother., 47: 2242-2248.

Poirel L, Pitout JD, Calvo L, Rodriguez-Martinez J, Church D, Nordmann $\mathrm{P}$ (2006). In vivo selection of fluoroqinolone-resistant Escherichia coli isolates expressing plasmid-mediated quinolone resistance and expanded-spectrum beta-lactamase. Antimicrob. Agents. Chemother., 50: 1525-1527.

Robicsek A, Jacoby GA, Hopper DC (2006a). The worldwide emergence of plasmid-mediated quinolone resistance. Lancet., 6: 629-640.

Robicsek A, Strahilevitz J, Sahm DF, Jacoby GA, Hooper DC (2006b). qnr revalence in ceftazidime-resistant Enterobacteriaceae isolates fromthe United States. Antimicrob. Agents. Chemother., 50: 2872-2874

Tran JH, Jacoby CA, Hooper DC (2005). Interaction of the plasmid-encoded quinolone resistance protein Qnr with Escherichia coli DNA gyrase. Antimicrob. Agents. Chemother., 49: 118-125. 